\title{
Research Communication LIF Upregulates Expression of NK-1R in NHBE Cells
}

\author{
Cheng-Ping Hu, ${ }^{1}$ Jun-Tao Feng, ${ }^{1}$ Yu-Ling Tang, ${ }^{1}$ Jin-Qi Zhu, ${ }^{2}$ Min-Juan Lin, ${ }^{1}$ and Ming-En Yu ${ }^{3}$ \\ ${ }^{1}$ Department of Respiratory Medicine, Xiangya Hospital of Central South University, Hunan Province, Changsha 410008, China \\ ${ }^{2}$ Department of Respiratory Medicine, Changsha Central Hospital, Hunan Province, Changsha 410008, China \\ ${ }^{3}$ Third Institute of Oceanography, SOA, Fujian Province, Xiamen 361005, China
}

Received 24 March 2006; Revised 30 July 2006; Accepted 7 August 2006

Leukemia inhibitory factor (LIF), a cytokine at the interface between neurobiology and immunology, is mainly mediated through JAK/STAT pathway and MAPK/ERK pathway. Evidence suggested LIF is related to the higher expression of neurokinin-1 receptor (NK-1R) in asthma. In this study, the immunohistochemistry stain showed the expressions of NK-1R, LIF, p-STAT3, and p-ERK1/2 in the lung tissues of allergic rats were increased compared with the controls, and the main positive cell type was airway epithelial cell. Normal human bronchial epithelial cells were treated with LIF in the presence or absence of AG490 (JAK2 inhibitor), PD98059 (MEK inhibitor), and the siRNA against STAT3. Western blot and RT-PCR indicated that LIF induced the expression of NK-1R, which was inhibited by the inhibitors mentioned above. No significant interaction was found between JAK/STAT pathway and MAPK/ERK pathway. In summary, bronchial epithelial cell changes in asthma are induced by LIF which promotes the expression of NK-1R, and JAK/STAT pathway and MAPK/ERK pathway may participate in this process.

Copyright (c) 2006 Cheng-Ping Hu et al. This is an open access article distributed under the Creative Commons Attribution License, which permits unrestricted use, distribution, and reproduction in any medium, provided the original work is properly cited.

\section{INTRODUCTION}

Leukemia inhibitory factor (LIF) is a pleiotrophic glycoprotein that belongs to the interleukin-6 (IL-6) cytokine family, which shares gp130 as the signal transducer. In the downstream of gp130, two important signal-transducing pathways have been recognized, the janus kinase/signal transducer and activator of transcription (JAK/STAT) pathway and the ras mitogen-activated protein kinase (MAPK) pathway [1-6]. There is widespread distribution of LIF within human lung tissue, where its physiological level is very low, but when exposed to proinflammatory cytokines such as IL- $1 \beta$, LIF gene expression upregulated [7]. In addition, high levels of LIF were also found in atopic patients and patients with diffuse pulmonary inflammation $[8,9]$.

Similar to the other neurotrophic factors such as nerve growth factor (NGF), it has been reported that LIF has been implicated in various processes of neuronal development, differentiation, survival and neurogenesis [10-12]. Furthermore, it was indicated that LIF could increase the expression of substance $P$ and its receptor (neurokinin-1 receptor; NK$1 \mathrm{R})$ both in mRNA and protein levels $[13,14]$. Substance $P$ and its receptor are main effective substances in airway neurogenic inflammation, Hu et al demonstrated that NGF upregulates NK-1R expression in normal rat lungs, and the expression of NK-1R increased in rat lungs which were in- fected with respiratory syncytial virus [15-17]. These data suggested that LIF has neuromodulatory role in the airways and may be an important signal molecule in the airway response to inflammation [18].

Bronchial epithelial cell is a barrier to airway structure, and it is an important target cell type in most respiratory diseases such as asthma. High levels of LIF and NK-1R were observed in bronchial epithelial cells of asthmatic rats [19]. However, whether the increased expression of NK-1R is related to LIF is unknown. If so, whether the role of LIF is mediated through JAK/STAT pathway and (or) MAPK pathway needs further investigation.

\section{MATERIALS AND METHODS}

\section{Animal preparation of asthmatic models}

Healthy male Sprague-Dawley rats, 6 to 8 weeks of age, were provided by the experimental animal center of Central South University. The animals were divided into 2 groups at random (asthmatic group and control group, $n=10$ ), and they were housed under specific pathogenfree conditions. Sensitization (the asthmatic group) was produced with an intraperitoneal injection of $100 \mathrm{mg}$ of chicken OVA(Sigma), $200 \mathrm{mg}$ of aluminum hydroxide(Sigma), and $5 \times 10^{9}$ heat-killed Bordetella pertussis (Wuhan Institute of 
Biological Products) in $1 \mathrm{ml}$ of sterile saline. The sham sensitization group (the control group) was treated by sterile saline intraperitoneal injection. Two weeks later, the rats in the asthmatic group were placed in a Plexiglas chamber $(20 \mathrm{~L})$ and challenged every day with 1\% OVA for $30 \mathrm{~min}$ using an ultrasonic nebulizer, while those in the control group received filtered air only. After a challenge peroid (10 days), the rats were killed by decapitation and bloodletting, and nonperfused excised lung tissues were fixed in $4 \%$ polyoxymethylene, then embedded in paraffin, and finally sliced into sections ( $5 \mu \mathrm{m}$ thick) for further study. The study protocol was in accordance with the guidelines for animal research and was approved by the Ethical and Research Committee of the hospital.

\section{Cell culture}

Normal human bronchial epithelial (NHBE) cells were obtained from the cell culture collection center of Yuantai Biosource (it was conducted in accordance with the declaration of Helsinki and the guidelines of the Ethical and Research Committee of the hospital). NHBE cells were cultured in Dulbecco's modified Eagle's medium supplemented with $10 \%$ fetal bovine serum, and cells were maintained at $37^{\circ}$ in a humidified atmosphere containing $5 \% \mathrm{CO}_{2}$. After $24 \mathrm{~h}$ in serum-free medium, cells were stimulated with recombinant human $\mathrm{LIF}$ (Chemicon) $(5 \mathrm{ng} / \mathrm{ml}, 30 \mathrm{~min}$ for detecting STAT3 and ERK1/2; $5 \mathrm{ng} / \mathrm{ml}, 24 \mathrm{~h}$ for detecting NK-1R) in pre-exposure or absence of AG-490 (JAK2 inhibitor, Biosource) (50 nmol/mL, $1 \mathrm{~h}$ ), PD-98059 (MEK inhibitor, Cell signaling technology) $(20 \mathrm{nmol} / \mathrm{mL}, 1 \mathrm{~h})$, PMA(ALEXIS Biochemicals) (10 ng/mL, $4 \mathrm{~h})$, and the small interfering RNA(siRNA) against STAT3(Genesil Biotechnology) $(2 \mu \mathrm{g} / \mathrm{mL}, 24 \mathrm{~h})$.

\section{Immunohistochemistry and immunocytochemistry}

Immunoreactivity for phospho-STAT3(p-STAT3), phosphoERK1/2(p-ERK1/2), NK-1R and LIF proteins were detected by streptavidin-biotin peroxidase complex method(SABC) (Boster Biotechnology). The primary antibodies against rat p-STAT3(rabbit polyclonal antibody, Santa Cruz Biotechnology), p-ERK1/2(mouse polyclonal antibody, Santa Cruz Biotechnology), NK-1R(rabbit polyclonal antibody, Novus Biologicals), and LIF(rabbit polyclonal antibody, Boster Biotechnology) were applied respectively. The secondary antibodies were affinity-purified biotinylated goat anti-rabbit(mouse) IgG (Boster Biotechnology). Nuclei were counterstained lightly with hematoxylin.

NHBE cells were plated at an approximate density of $2 \times 10^{5}$ cells $/ \mathrm{cm}^{2}$ onto tissue culture chamber slides (Lab Tek, Tokyo) in medium containing 10\% FBS. After $24 \mathrm{~h}$, cells were replenished with serum-free medium and incubated for $24 \mathrm{~h}$. NHBE cells were preincubated with or without AG-490, PD98059, PMA, or the siRNA against STAT3 and then stimulated with LIF. Following this, the cells were fixed in $4 \%$ polyoxymethylene, and stained with primary antibody against NK-1R. Primary antibody was detected by affinity-purified biotinylated secondary antibody (Boster Biotechnology), and the stained cells were observed by microscopy. Ten fields $(\times 200)$ were chosen at random under microscope, and a positive cell ratio was obtained by counting (positive cells number/total cells number).

\section{RNA interference}

The siRNA oligonucleotide sequences against STAT3 (NM_139276.2) were in accordance with Lee et al and Konnikova et al [20, 21], siRNA-1: 5'-GATCCGCATCTGCCTAGATCGGCTATTCAAGACGTAGCCGATCTAGGCAGATGTTTTTTGAATTCA-3', , 3'-GCGTAGACGGATCTAGCCGATAAGTTCTGCATCGGCTAGATCCGTCTACAAAAAACTTAAGTTCGA-5'; siRNA-2: 5'-GATCCGTGTTCTCTATCAGCACAATTTCAAGACGATTGTGCTGATAGAGAACATTTTTTGAATTCA-3', 3'-GCACAAGAGATAGTCGTGTTAAAGTTCTGCTAACACGACTATCTCTTGTAAAAAACTTAAGTTCGA-5; negative control siRNA: 5' -GATCCGACTTCATAAGGCGCATGCTTCAAGACGGCATGCGCCTTATGAAGTCTTTTTTGTCGACA-3', 3'-GCTGAAGTATTCCGCGTACGAAGTTCTGCCGTACGCGGAATACTTCAGAAAAAACAGCTGTTCGA-5' (synthesized by Genesil Biotechnology). The siRNAs $(2 \mu \mathrm{g} / \mathrm{mL})$ were introduced into cells using the METAFECTENE highly efficient transfection reagent (Biontex) according to the manufacturer's instructions.

\section{Western blot}

Cells were harvested in lysis buffer, and the lysates were cleared by centrifugation at $12000 \mathrm{~g}$ for $10 \mathrm{~min}$ at $4^{\circ}$. The proteins were separated by $10 \%$ SDS-PAGE, and transferred to polyvinylidene difluoride membranes, then probed with primary antibodies against human STAT3, p-STAT3(tyr) (rabbit polyclonal antibody, Santa Cruz Biotechnology), ERK1/2, p-ERK1/2 (mouse polyclonal antibody, Santa Cruz Biotechnology), GAPDH (rabbit polyclonal antibody, Santa Cruz Biotechnology). The primary antibodies that bounded to the target proteins were detected using horseradish peroxidase-conjugated anti-rabbit IgG(Promega), or antimouse IgG(Cortex Biochem), as appropriate. The antibodies were visualized with enhanced chemiluminescent detection (Pierce Biotechnology). The intensity of target bands was corrected by GAPDH calculated with the FluorChem 8900 software system.

\section{RT-PCR}

Total RNAs were extracted using TRIZOL(Invitrogen). cDNAs were synthesized using the RevertAid H Minus First Strand cDNA Synthesis Kit (Fermentas) with oligo(dT)18 primers. The primer sequences used were as follows. Human NK-1R: forward primer 5' GGGACTCCTCTGACCGCTAC $3^{\prime}$, reverse primer $5^{\prime}$ TCCAGGCGGCTGACTTTGTA 3', PCR product 376 bp (753-1128); human $\beta$-actin: forward primer 5' CCTTCCTGGGCATGGAGTC $3^{\prime}$, reverse primer 5' GAGGAGCAATGATCTTGATCTTC 3', PCR product 


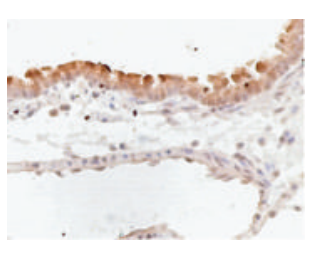

(a)

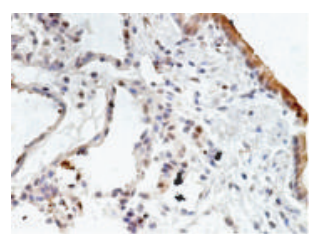

(e)

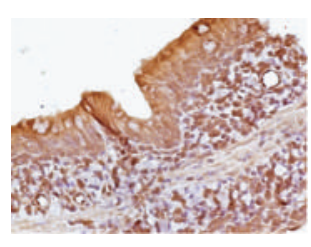

(b)

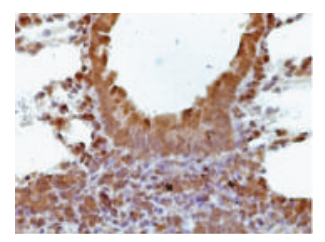

(f)

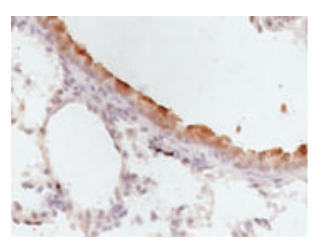

(c)

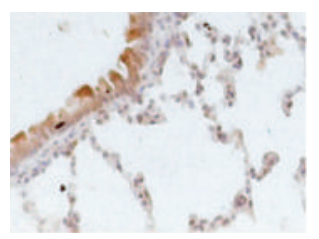

(g)

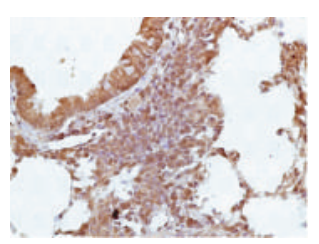

(d)

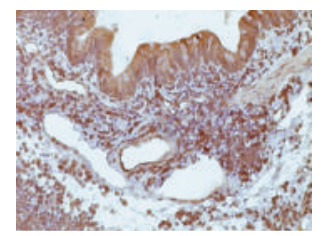

(h)

FIGURE 1: Expression of NK-1R, LIF, p-STAT3, and p-ERK1/2 in lung tissues of asthmatic rats (SABC $\times 200)$. Immunohistochemistry was performed on lung tissue of asthmatic rats. There were higher expressions for NK-1R ((a) control group, (b) asthmatic group) and LIF ((c) control group, (d) asthmatic group) in the asthmatic rats than those in the control rats, and the similar changes were observed for p-STAT3 ((e) control group, (f) asthmatic group) and p-ERK1/2 ((g) control group, (h) asthmatic group). The main positive cell type was airway epithelial cell.

204 bp (867-1070). RT-PCR analysis was performed as described by $\mathrm{Hu}$ et al with minor modifications [15]. The intensity of target mRNA levels was corrected by $\beta$-actin transcripts calculated with the FluorChem 8900 software system.

\section{Statistical analysis}

The data presented in (Figures 1, 2, 3, 4, and 5) were expressed as means $\pm \mathrm{SD}(n=3)$. Statistical significance among mean values was evaluated with an ANOVA. Differences were considered significant when $P<.05$.

\section{RESULTS}

\section{Expression of LIF, NK-1R, p-STAT3, and p-ERK1/2 in lung tissues of asthmatic rats}

Immunohistochemistry was performed in lung tissues of rats, and it indicated a higher expression of LIF in the asthmatic rats compared to that in the control group. Consistent with that, similar changes were observed for NK-1R, pSTAT3, and p-ERK1/2. The main positive cell type was airway epithelial cell, and other positive types were also observed such as lymphocyte (Figure 1).

\section{Effects of AG-490, PD-98059, or PMA on LIF-induced activation of signal transduction and activation of transcription (STAT3) and ERK1/2}

Western blot was performed on cells that had been preincubated with or without AG-490, PD-98059, or PMA and then stimulated with LIF. LIF induced activation of tyrosine phosphorylation of STAT3 (versus the control cells, $P<.01$ ), and tyrosine phosphorylation of STAT3 was inhibited by AG490 (the cells stimulated with LIF in the presence of AG490 versus the cells stimulated with LIF, $P<.01$ ), but not by PD-98059 (the cells stimulated with LIF in the presence of PD-98059 versus the cells stimulated with LIF, $P>.05$ ), and the results also indicated phosphorylation of STAT3 that was not affected by PMA (Figure 2(a)). Nevertheless, the expression of total-STAT3 was not affected by the factors mentioned above (Figure 2(b)). LIF induced activation of phosphorylation of ERK1/2 (versus the control cells, $P<.01$ ), and ERK1/2 activation was inhibited by PD-98059 (the cells stimulated with LIF in the presence of PD-98059 versus the cells stimulated with LIF, $P<.01$ ), but not by AG-490 (the cells stimulated with LIF in the presence of AG-490 versus the cells stimulated with LIF, $P>.05$ ) (Figure 2(c)). Similar to that of total-STAT3, the expression of total-ERK1/2 did not change (Figures 2(d), 2(e)). PMA increased the expression of p-ERK1/2 in NHBE cells (versus the control cells, $P<.01$ ), but there was no significant difference between the cells stimulated with LIF and the cells stimulated with LIF in the presence of PMA.

\section{Effects of AG-490, PD-98059, or PMA on LIF-induced expression of NK-1R}

Immunocytochemistry and RT-PCR were performed on cells that had been preincubated with or without AG-490, PD98059, or PMA and then stimulated with LIF. LIF induced expression of NK-1R in NHBE cells (versus the control cells, $P<.01$ ), both AG-490 and PD-98059 suppressed the LIFinduced expression of NK-1R by $58 \%$ (the cells stimulated with LIF in the presence of AG- 490 versus the cells stimulated with LIF, $P<.01$ ) and $60 \%$ (the cells stimulated with 


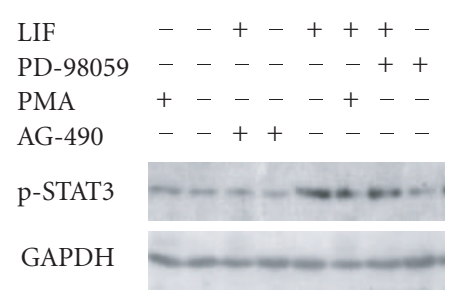

(a)

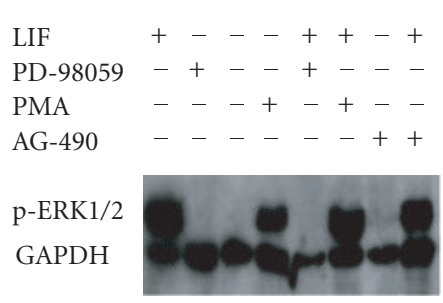

(c)

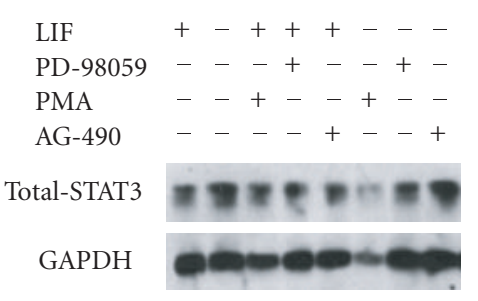

(b)

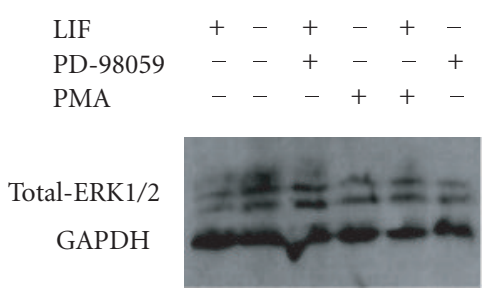

(d)

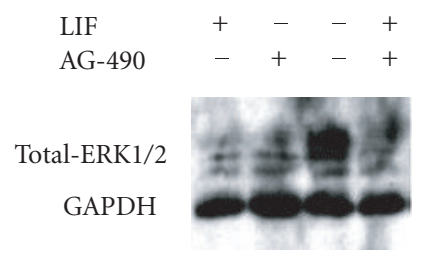

(e)

FIGURE 2: Effects of AG-490, PD-98059, or PMA on LIF-induced activation of signal transduction and activation of transcription_(STAT3) and ERK1/2. Western blot was performed on NHBE cells that had been preincubated with or without AG-490, PD-98059, or PMA and then stimulated with LIF. (a) LIF induced activation of tyrosine phosphorylation of STAT3, and tyrosine phosphorylation of STAT3 was inhibited by AG-490, but not by PD-98059, and not affected by PMA. (b) LIF did not enhance the expression of total-STAT3, and its expression was not affected by AG-490, PD-98059, and PMA. (c) LIF induced activation of phosphorylation of ERK1/2, and ERK1/2 activation was inhibited by PD-98059, but not by AG-490; PMA increased the expression of p-ERK1/2 in NHBE cells, but there were no significant differences between the cells stimulated with LIF and the cells stimulated with LIF in the presence of PMA. (d) and (e) LIF did not enhance the expression of total-ERK1/2, furthermore, AG-490, PD-98059, and PMA also did not affect it. Experiments were repeated three times with similar results, and the data was expressed as the mean ratio (target/GAPDH) \pm SD.

LIF in the presence of PD-98059 versus the cells stimulated with LIF, $P<.01)$; on the contrary, PMA increased the expression of NK-1R in NHBE cells (Figures 3 and 4(a)).

\section{Effects of siRNA(STAT3) on LIF-induced activation of signal transduction and activation of transcription (STAT3) and ERK1/2}

Western blot was performed on cells that had been preincubated with or without siRNA(STAT3) and then stimulated with LIF. LIF-induced tyrosine phosphorylation of STAT3 was inhibited by siRNA-1 and siRNA-2, and the inhibition ratio was $85 \%$ and $42 \%$, respectively (Figure $5(\mathrm{a})$ ). In addition, the expression of total-STAT3 was also inhibited by siRNA-1 and siRNA-2 (56\% and 37\%, resp) (Figure 5(b)). Because of the higher inhibition ratio, siRNA-1 was later chosen for interference in the cells. However, siRNA-1 did not affect the expression of $\mathrm{p}$-ERK1/2 and total-ERK1/2 (Figures $5(\mathrm{c}), 5(\mathrm{~d}))$.

\section{Effects of siRNA(STAT3) on LIF-induced expression of $\mathrm{NK}-1 \mathrm{R}$}

Immunocytochemistry and RT-PCR were performed on cells that had been preincubated with or without siRNA-1 (STAT3) and then stimulated with LIF. The LIF-induced expression of NK-1R was inhibited by siRNA-1 both at the mRNA level and the protein level (the cells stimulated with LIF in the presence of siRNA-1 versus the cells stimulated with LIF, $P<.01$ ), but it was not affected by negative control siRNA and sham plasmid (the cells stimulated with LIF in the presence of control siRNA or sham plasmid versus the cells stimulated with LIF, $P>$.05) (Figures 3 and $4(\mathrm{~b})$ ).

\section{DISCUSSION}

LIF is a cytokine at the interface between neurobiology and immunology [22]. Exposure of neural tissue to proinflammatory cytokines, such as IL- $1 \beta$ or injury, increases the 


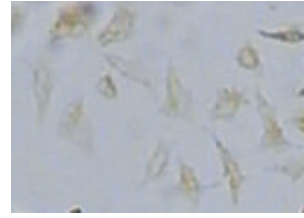

(a)

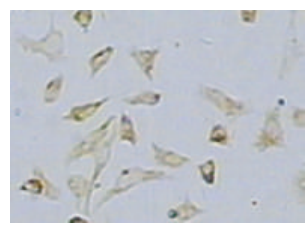

(e)

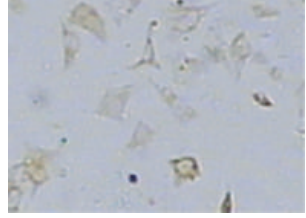

(b)

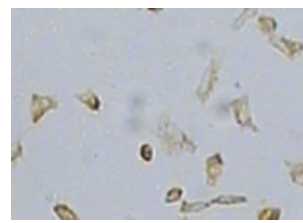

(f)

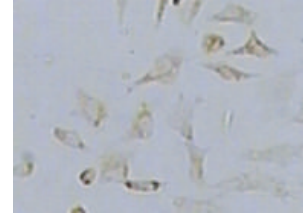

(c)

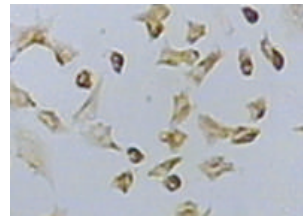

(g)

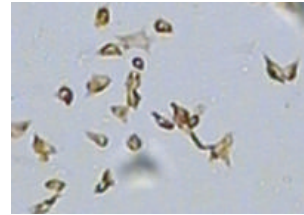

(d)

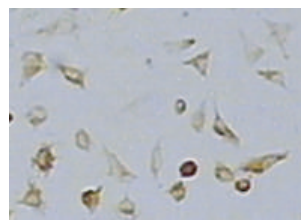

(h)

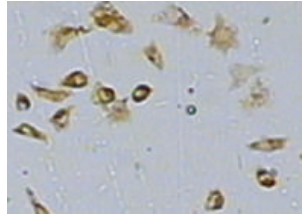

(i)

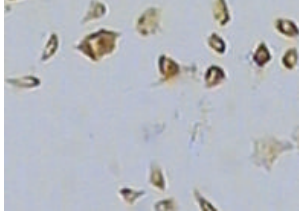

(j)

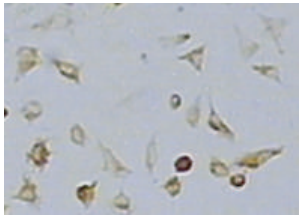

(k)

FIgure 3: Effects of AG-490, PD-98059, PMA, or siRNA-1(STAT3) on LIF-induced expression of NK-1R detected by immunocytochemistry $(S A B C \times 200)$. Immunocytochemistry was performed on cells that had been preincubated with or without AG-490, PD-98059, PMA, or siRNA-1(STAT3) and then stimulated with LIF ( a) control, (b) PD-98059, (c) AG-490, (d) LIF, (e) PMA, (f) LIF + PD-95059, (g) LIF + PMA, (h) LIF + AG-490, (i) LIF + control siRNA, (j) LIF + sham plasmid, (k) LIF + siRNA-1 against STAT3). LIF induced expression of NK-1R, which was inhibited by AG-490, PD-98059, and siRNA-1 against STAT3, but was affected neither by the control siRNA nor the sham plasmid. Experiments were repeated three times with similar results, and the data was expressed as the mean ratio (positive cells number/total cells number) \pm SD.

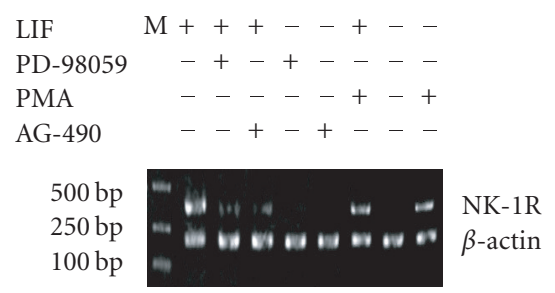

(a)

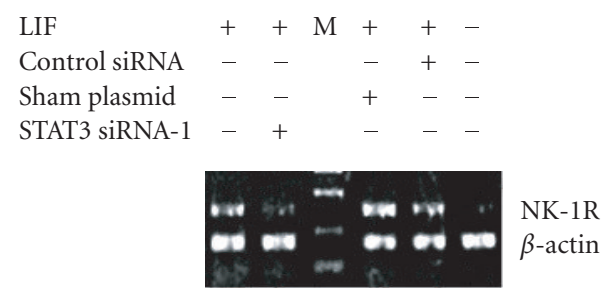

(b)

FIGURE 4: Effects of AG-490, PD-98059, PMA, or siRNA-1(STAT3) on LIF-induced expression of NK-1R detected by RT-PCR. RT-PCR was performed on cells that had been preincubated with or without AG-490, PD-98059, PMA, or siRNA-1(STAT3) and then stimulated with LIF. (a) LIF induced expression of NK-1R mRNA, and that was inhibited by AG-490 and PD-98059; PMA increased the expression of NK-1R mRNA in NHBE cells. (b) LIF-induced expression of NK-1R mRNA was inhibited by siRNA-1 against STAT3, but was affected neither by the control siRNA nor the sham plasmid. Experiments were repeated three times with similar results, and the data was expressed as the mean ratio $(\operatorname{target} / \beta$-actin $) \pm \mathrm{SD}$.

synthesis and release of LIF, which in turn increases mRNA and protein of substance $P$ and its receptor (NK-1R). Similarly, LIF also can induce neuropeptide synthesis and release in neurons that do not normally produce neuropeptides, and these studies suggested that LIF has an important neuroimmune linkage function $[13,14,23]$. LIF dose dependently augmented eosinophil migration and other functions in response to substance $P$, resulting in bidirectional interactions between inflammatory cells and nerves in allergic diseases [8].

It was found that serum LIF levels were higher in atopic patients with mild asthma than in nonatopic normal donors [8]. Consistent with that, high levels of LIF were also found in bronchoalveolar lavage fluid obtained from patients with the acute respiratory distress syndrome, in which there was diffuse pulmonary inflammation [9]. Immunohistochemistry 


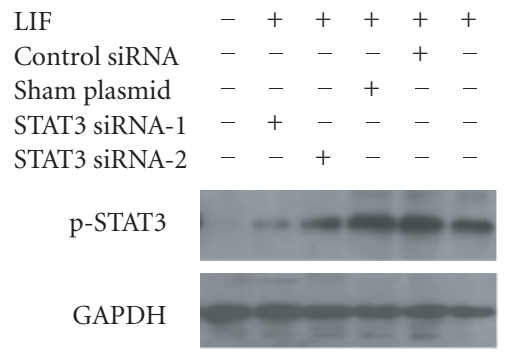

(a)

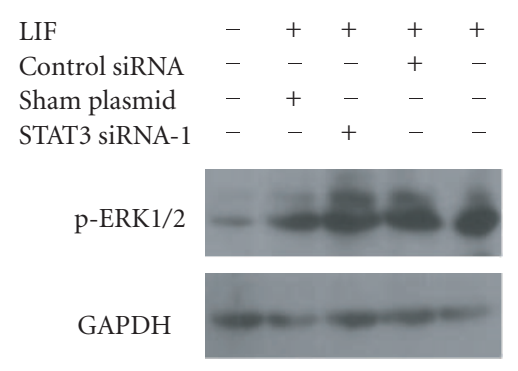

(c)

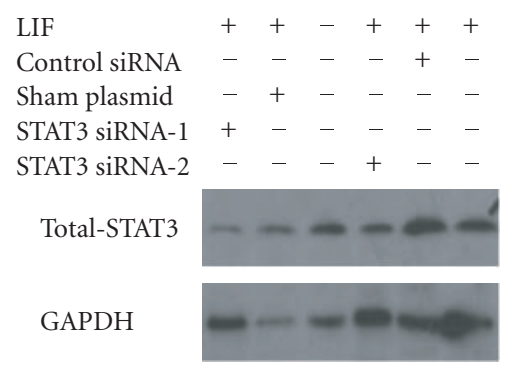

(b)

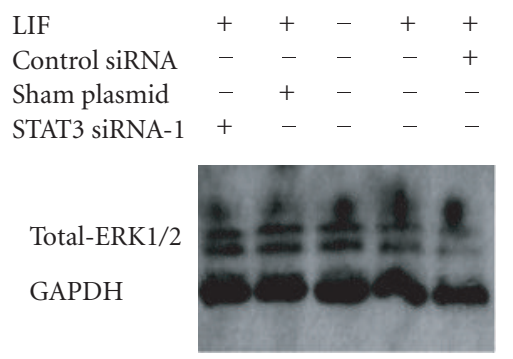

(d)

FIGURE 5: Effects of siRNA(STAT3) on LIF-induced activation of signal transduction and activation of transcription (STAT3) and ERK1/2. Western blot was performed on NHBE cells that had been preincubated with or without siRNA(STAT3) and then stimulated with LIF. (a) LIF induced activation of tyrosine phosphorylation of STAT3, and tyrosine phosphorylation of STAT3 was inhibited by siRNA-1 and siRNA-2, but neither by control siRNA nor sham plasmid. (b) Similiar to phosphorylation of STAT3, total-STAT3 was inhibited by siRNA1 and siRNA-2, but neither by control siRNA nor sham plasmid. (c) LIF induced activation of phosphorylation of ERK1/2, but ERK1/2 activation was not inhibited by siRNA-1 against STAT3. (d) Similiar to phosphorylation of ERK1/2, the expression of total-ERK1/2 was not affected by siRNA-1 against STAT3. Experiments were repeated three times with similar results, and the data was expressed as the mean ratio(target/GAPDH) $\pm \mathrm{SD}$.

demonstrated the presence of LIF in epithelial cells, mesenchymal cells, and nerve fibers in the human airway, and it was found that these airway structural cell types release LIF and its receptor (LIFR) in response to inflammatory stimuli such as proinflammatory cytokines. Subsequently, LIF augmented contractile responses to tachykinins in airway explants $[7,24]$.

In animal models, it was indicated that NK-1R was involved in the development of allergen-induced airway hyperreactivity to histamine after both the early asthmatic reactions and late asthmatic reactions, and NK-1R-mediated inflammation of airways may contribute to this process [25]. In the present study, the airway tissues of asthmatic rats were tested by immunohistochemistry, and it was found that LIF expression in the asthmatic rats was higher than that in the normal control group, at the same time, NK-1R expression in the asthmatic rats also increased.

Incubation of rat sympathetic cervical ganglia with LIF downregulated the expression of muscarinic $\mathrm{M}_{2} \mathrm{R}$ mRNA, while at the same time increased the expression of substance $P$ and NK-1R $[13,14]$. After incubation of tracheal explants with LIF, there were no observable increases of substance $P$ expression compared to the control, however, measurement of NK-1R expression was not done [18]. Substance $P$ is a member of tachykinin family, which is usually not steadily expressed, and is not easily detected. As a ligand, substance $P$ can express biological effect on the condition of combining with its specific receptor NK-1R, and thus, the biological activity of substance $P$ would be reflected by detecting the expression of NK-1R.

LIF is a pleiotrophic glycoprotein that belongs to the IL6 cytokine family, which shares the common gp130 receptor chain as the signal transducing protein. LIF signaling is mediated through the LIF receptor which heterodimerizes with the gp130 receptor upon LIF binding [5]. Activation of the LIFR- $\beta$-gp130 heterodimer results in the rapid activation of janus kinases (JAKs) which in turn phosphorylate tyrosine residues of LIFR- $\beta$ and gp130 $[3,4,6]$. These phosphorylated tyrosine residues form docking sites for signaling molecules including STAT3 and SHP2 [4]. STATs are transcription factors, which form dimers upon phosphorylation of a specific tyrosine residue that is located in a conserved SHP2 domain [26]. STAT dimerization allows nuclear translocation and the transcriptional activation of target genes [26]. SHP2 is a tyrosine phosphatase which signals upstream of the Ras/MAP kinase signal transduction pathway [4, 27-29]. As a result, downstream of the pathway molecules such as ERK1/2 and p38 is activated sequentially. 
As the important pathways in eukaryocyte, Ras/MEK/ ERK cascade pathway and JAK/STAT cascade pathway may be closely interrelated. In many cell types in culture, sustained expression of activated Ras or its downstream effector can elicit cell cycle arrest and differentiation, and some reseachers revealed that the biological effects of the Ras/Raf/ MEK/ERK pathway were activated via LIF/JAK/STAT pathway [30-32]. However, in LIF/gp130-mediated cardiac hypertrophy, AG490 (JAK2 inhibitor) and PD98059 (a specific MEK inhibitor) were applied to compare the significance between ERK cascade and JAK/STAT cascade, and it was shown that LIF-induced expression of c-fos and others was markedly suppressed by PD98059 and moderately suppressed by AG490, but STAT3 activation was not suppressed by PD98059 and ERK1/2 activation was not suppressed by AG490 [33]. It was suggested that the two pathways are independent of each other.

In the present study, the airway tissues of asthmatic rats were tested for LIF-linked substance by immunohistochemistry. Compared with the control, there were increased expressions for LIF and NK-1R in the asthmatic rats, and similar changes were observed for p-STAT3 and p-ERK1/2. The main positive cell type was airway epithelial cell, and other types were also observed such as lymphocyte and structural cells. These results were similar to the data provided by Knight et al [24] and Bai et al [34]. Combining these findings with the data mentioned above, it is hypothesized that LIF enhances the NK-1R expression in airway of asthmatic models, and the enhancement may be connected with the JAK-STAT pathway or the MAPK pathway. Furthermore, airway epithelial cells may be the main effective cell type in this process.

To test the hypothesis, we cultured NHBE cells treated with LIF, inhibitors of the JAK/STAT and MAPK/ERK pathways (AG490 and PD98059), and the activator of protein kinase C(PMA). This study demonstrated that LIF induced expression of NK-1R in NHBE cells, which was determined by RT-PCR and immunocytochemistry. In this process, similar to NK-1R, the expressions of p-STAT3 and p-ERK1/2 in NHBE cells treated with LIF all increased. Expressions of total-STAT3 and total-ERK1/2 between LIF-treated cells and the control cells were not significantly different. AG490 and PD98059 suppressed the LIF-induced expression of NK1R. AG490 inhibited the LIF-induced phosphorylation of STAT3, whereas PD98059 showed no inhibition; on the contrary, PD98059 clearly inhibited the LIF-induced phosphorylation of ERK1/2, whereas AG490 showed no inhibition. PMA, a potent activator of protein kinase $C$, is considered to have a strong effect to activate ERK1/2, and further increase the levels of related substances in the downsteam of ERK pathway (eg, c-fos and c-jun) [35]. Our study showed that, compared with the control, PMA increased the expressions of p-ERK1/2 and NK-1R in NHBE cells; but there was no significant difference between the cells stimulated with LIF in the presence of PMA and the cells stimulated with LIF. These findings indicated that the JAK/STAT pathway and the MAPK pathway play different roles in LIF-induced expression of NK-1R in NHBE cells, and suggest that these path- ways may be independent in producing a marked biological effect.

To further confirm the results mentioned above, we designed this study to block STAT3 expression by siRNA. It was found that the siRNA against STAT3 specifically reduced STAT3 expression in LIF-induced NHBE cells. For the blockade of STAT3, the LIF-induced expression of NK-1R also decreased, whereas the expression of ERK1/2 (p-ERK1/2 and total-ERK1/2) did not change in this process.

In conclusion, we have demonstrated that NK-1R expression is upregulated in NHBE cells when exposed to LIF, and this process may be mediated by JAK2/STAT3 pathway and ERK1/2 pathway, but no observable interaction was found between the two pathways in the present study. Since signaling cascades often converge from multiple upstream mediators, it is possible that the cross-talk and alternative pathways exist. Thus, whether these factors influenced our results further investigation is required.

\section{ACKNOWLEDGMENT}

This work was supported by National Natural Scientific Foundation of China (no. 30300146).

\section{REFERENCES}

[1] Jo C, Kim H, Jo I, et al. Leukemia inhibitory factor blocks early differentiation of skeletal muscle cells by activating ERK. Biochimica et Biophysica Acta - Molecular Cell Research. 2005;1743(3):187-197.

[2] Schiemann WP, Bartoe JL, Nathanson NM. Box 3-independent signaling mechanisms are involved in leukemia inhibitory factor receptor $\alpha$ - and gp130-mediated stimulation of mitogen-activated protein kinase. Evidence for participation of multiple signaling pathways which converge at Ras. Journal of Biological Chemistry. 1997;272(26):16631-16636.

[3] Schuringa JJ, van der Schaaf S, Vellenga E. LIF-induced STAT3 signaling in murine versus human embryonal carcinoma (EC) cells. Experimental Cell Research. 2002;274(1):119-129.

[4] Heinrich PC, Behrmann I, Muller-Newen G, Schaper F, Graeve L. Interleukin-6-type cytokine signalling through the gp130/Jak/STAT pathway. Biochemical Journal. 1998;334 (2):297-314.

[5] Gearing DP, Comeau MR, Friend DJ, et al. The IL-6 signal transducer, gp130: an oncostatin $\mathrm{M}$ receptor and affinity converter for the LIF receptor. Science. 1992;255(5050):14341437.

[6] Stahl N, Boulton TG, Farruggella T, et al. Association and activation of Jak-Tyk kinases by CNTF-LIF-OSM-IL-6 $\beta$ receptor components. Science. 1994;263(5143):92-95.

[7] Knight DA, Lydell CP, Zhou D, Weir TD, Schellenberg RR, Bai TR. Leukemia inhibitory factor (LIF) and LIF receptor in human lung distribution and regulation of LIF release. American Journal of Respiratory Cell and Molecular Biology. 1999;20(4):834-841.

[8] Zheng X, Knight DA, Zhou D, et al. Leukemia inhibitory factor is synthesized and released by human eosinophils and modulates activation state and chemotaxis. Journal of Allergy and Clinical Immunology. 1999;104(1):136-144.

[9] Jorens PG, De Jongh R, Bossaert LL, et al. High levels of leukaemia inhibitory factor in ARDS. Cytokine. 1996;8(11): 873-876. 
[10] Kim EJ, Simpson PJ, Park D-J, Liu BQ, Ronnett GV, Moon C. Leukemia inhibitory factor is a proliferative factor for olfactory sensory neurons. NeuroReport. 2005;16(1):25-28.

[11] Yamamori T, Fukada K, Aebersold R, Korsching S, Fann M-J, Patterson $\mathrm{PH}$. The cholinergic neuronal differentiation factor from heart cells is identical to leukemia inhibitory factor. Science. 1989;246(4936):1412-1416.

[12] Murphy M, Reid K, Brown MA, Bartlett PF. Involvement of leukemia inhibitory factor and nerve growth factor in the development of dorsal root ganglion neurons. Development. 1993;117(3):1173-1182.

[13] Ludlam WH, Chandross KJ, Kessler JA. LIF-and IL-1 $\beta$ mediated increases in substance $\mathrm{P}$ receptor mRNA in axotomized, explanted or dissociated sympathetic ganglia. Brain Research. 1995;685(1-2):12-20.

[14] Ludlam WH, Zang Z, McCarson KE, Krause JE, Spray DC, Kessler JA. mRNAs encoding muscarinic and substance $\mathrm{P}$ receptors in cultured sympathetic neurons are differentially regulated by LIF or CNTF. Developmental Biology. 1994;164(2):528-539.

[15] Hu C-P, Wedde-Beer K, Auais A, Rodriguez MM, Piedimonte G. Nerve growth factor and nerve growth factor receptors in respiratory syncytial virus-infected lungs. American Journal of Physiology - Lung Cellular and Molecular Physiology. 2002;283(2):L494-L502.

[16] King KA, Hu C-P, Rodriguez MM, Romaguera R, Jiang X, Piedimonte G. Exaggerated neurogenic inflammation and substance $\mathrm{P}$ receptor upregulation in RSV-infected weanling rats. American Journal of Respiratory Cell and Molecular Biology. 2001;24(2):101-107.

[17] Piedimonte G. Contribution of neuroimmune mechanisms to airway inflammation and remodeling during and after respiratory syncytial virus infection. Pediatric Infectious Disease Journal. 2003;22(suppl 2):S66-S75.

[18] Knight DA, D’Aprile AC, Spalding LJ, Goldie RG, Thompson PJ. Leukaemia inhibitory factor (LIF) upregulates excitatory non-adrenergic non-cholinergic and maintains cholinergic neural function in tracheal explants. British Journal of Pharmacology. 2000;130(5):975-979.

[19] Lin M-J, Lao XJ, Ma HM, Tang Y-L. The relationship between leukemia inhibitory factor and neurokinin receptors in a rat model of asthma [in Chinese]. Zhonghua Jie He He Hu Xi Za Zhi. 2005;28(12):820-824.

[20] Lee SO, Lou W, Qureshi KM, Mehraein-Ghomi F, Trump DL, Gao AC. RNA interference targeting STAT3 inhibits growth and induces apoptosis of human prostate cancer cells. The Prostate. 2004;60(4):303-309.

[21] Konnikova L, Kotecki M, Kruger MM, Cochran BH. Knockdown of STAT3 expression by RNAi induces apoptosis in astrocytoma cells. BMC Cancer. 2003;3(1):23-31.

[22] Patterson PH. Leukemia inhibitory factor, a cytokine at the interface between neurobiology and immunology. Proceedings of the National Academy of Sciences of the United States of America. 1994;91(17):7833-7835.

[23] Kessler JA, Freidin MM, Kalberg C, Chandross KJ. Cytokines regulate substance $\mathrm{P}$ expression in sympathetic neurons. Regulatory Peptides. 1993;46(1-2):70-75.

[24] Knight DA, McKay K, Wiggs B, Schellenberg RR, Bai TR. Localization of leukaemia inhibitory factor to airway epithelium and its amplification of contractile responses to tachykinins. British Journal of Pharmacology. 1997;120(5):883-891.

[25] Schuiling M, Zuidhof AB, Zaagsma J, Meurs H. Involvement of tachykinin NK1 receptor in the development of allergeninduced airway hyperreactivity and airway inflammation in conscious, unrestrained guinea pigs. American Journal of Respiratory and Critical Care Medicine. 1999;159(2):423-430.

[26] Schindler C, Darnell JE Jr. Transcriptional responses to polypeptide ligands: the JAK-STAT pathway. Annual Review of Biochemistry. 1995;64:621-651.

[27] Fukada T, Hibi M, Yamanaka Y, et al. Two signals are necessary for cell proliferation induced by a cytokine receptor gp130: involvement of STAT3 in anti-apoptosis. Immunity. 1996;5(5):449-460.

[28] Nishida K, Yoshida Y, Itoh M, et al. Gab-family adapter proteins act downstream of cytokine and growth factor receptors and T- and B-cell antigen receptors. Blood. 1999;93(6):18091816.

[29] Takahashi-Tezuka M, Yoshida Y, Fukada T, et al. Gab1 acts as an adapter molecule linking the cytokine receptor gp130 to ERK mitogen-activated protein kinase. Molecular and Cellular Biology. 1998;18(7):4109-4117.

[30] Park J-I, Strock CJ, Ball DW, Nelkin BD. The Ras/Raf/MEK/ extracellular signal-regulated kinase pathway induces autocrine-paracrine growth inhibition via the leukemia inhibitory factor/JAK/STAT pathway. Molecular and Cellular Biology. 2003;23(2):543-554.

[31] Park J-I, Strock CJ, Ball DW, Nelkin BD. Interleukin-1 $\beta$ can mediate growth arrest and differentiation via the leukemia inhibitory factor/JAK/STAT pathway in medullary thyroid carcinoma cells. Cytokine. 2005;29(3):125-134.

[32] Park J-I, Powers JF, Tischler AS, Strock CJ, Ball DW, Nelkin BD. GDNF-induced leukemia inhibitory factor can mediate differentiation via the MEK/ERK pathway in pheochromocytoma cells derived from $n f 1$-heterozygous knockout mice. Experimental Cell Research. 2005;303(1):79-88.

[33] Kodama H, Fukuda K, Pan J, et al. Significance of ERK cascade compared with JAK/STAT and PI3-K pathway in gp130mediated cardiac hypertrophy. American Journal of Physiology - Heart and Circulatory Physiology. 2000;279(4):H1635H1644.

[34] Bai TR, Zhou D, Weir T, et al. Substance P (NK1)- and neurokinin A (NK2)-receptor gene expression in inflammatory airway diseases. American Journal of Physiology. 1995;95(269): L309-L317.

[35] Deng X-F, Rokosh DG, Simpson PC. Autonomous and growth factor-induced hypertrophy in cultured neonatal mouse cardiac myocytes: comparison with rat. Circulation Research. 2000;87(9):781-788. 


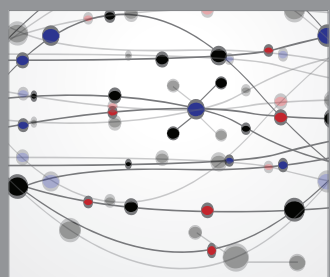

The Scientific World Journal
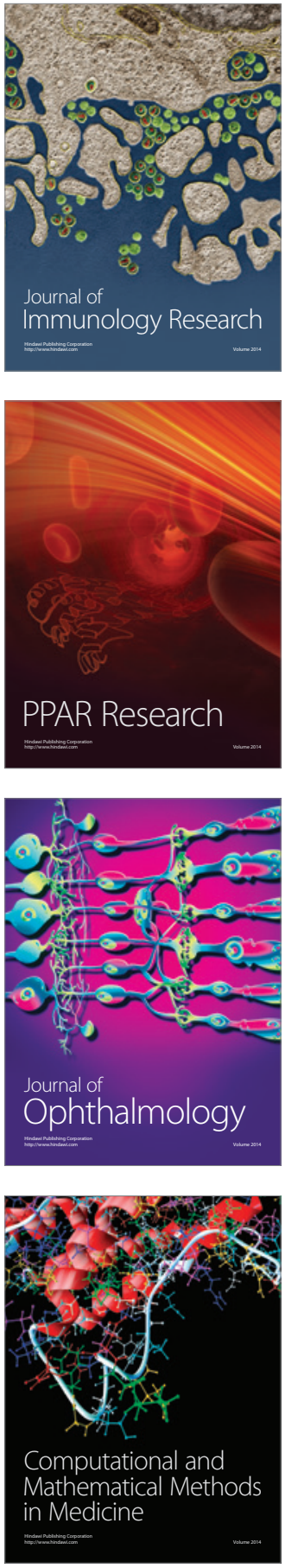

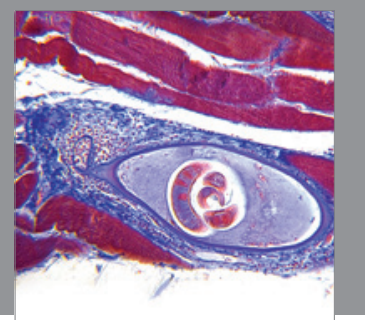

Gastroenterology

Research and Practice
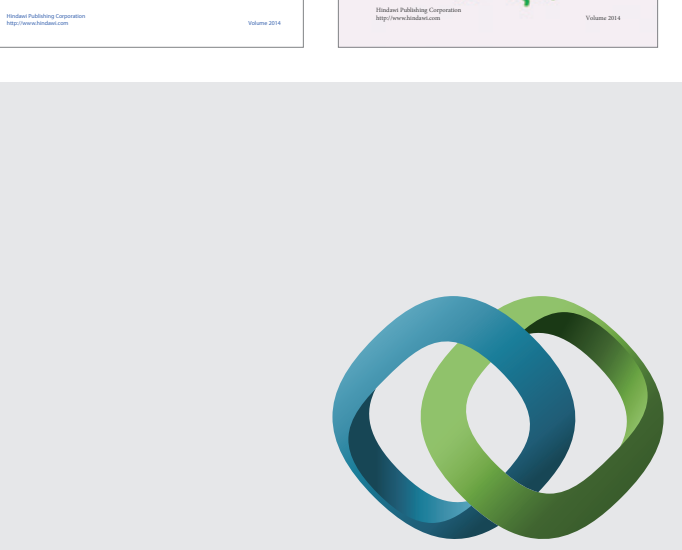

\section{Hindawi}

Submit your manuscripts at

http://www.hindawi.com
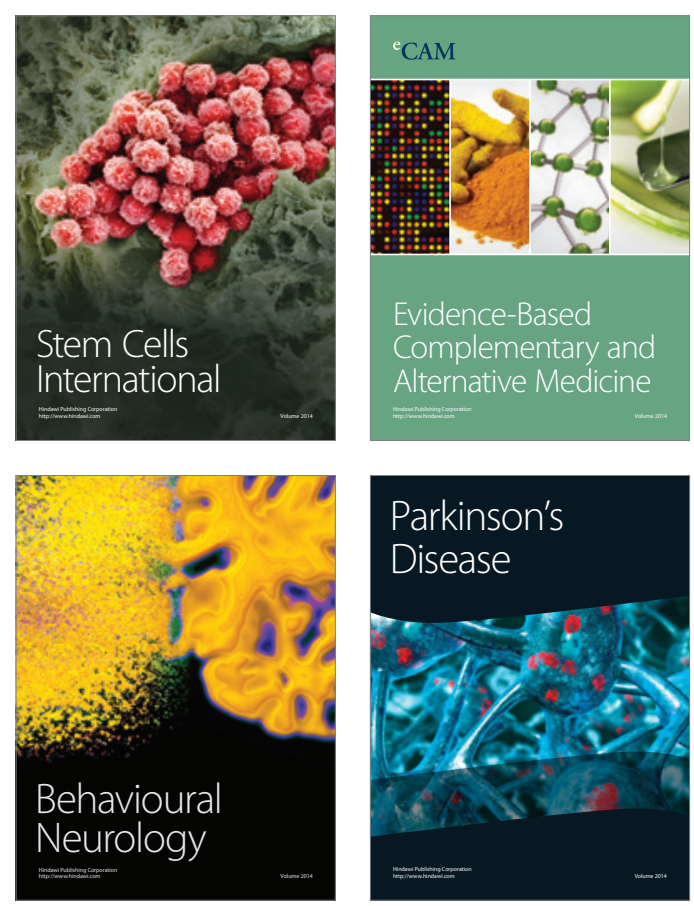

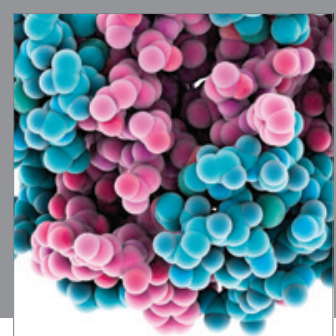

Journal of
Diabetes Research

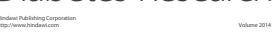

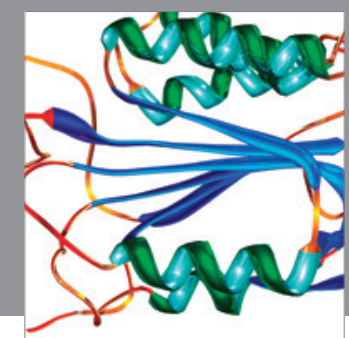

Disease Markers
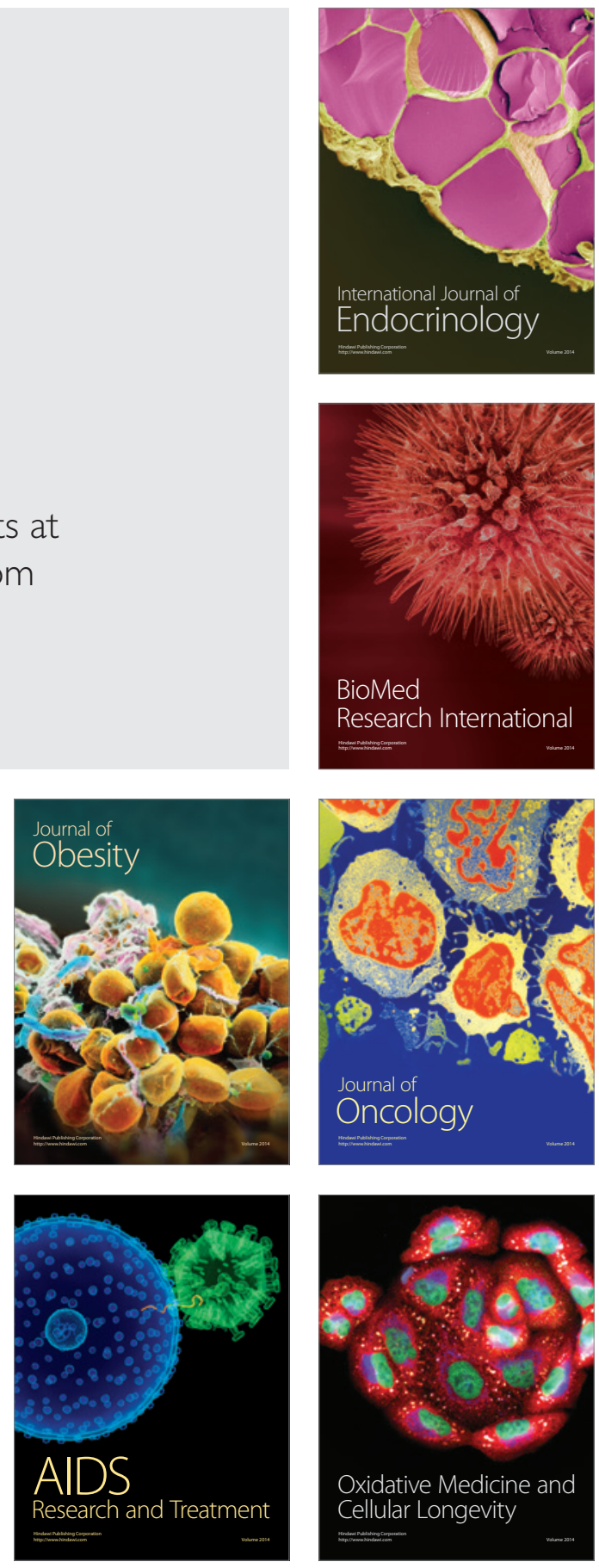